\title{
Synthesis and Characterization of PPY-PANI- ZnO Hybrid Nanocomposites for OLEDs Applications as Electron Transport Layer
}

\author{
Rohit Kandulna, Ram B. Choudhary*, Rajinder Singh \\ Nanostructured Composite Materials Laboratory, Department of Applied Physics, Indian Institute of Technology \\ (IIT-ISM), Dhanbad 826004, India
}

*Corresponding author: E-mail: rbchec@yahoo.co.in; Tel: (+91) 9471191381

DOI: 10.5185/amp.2019.7004

www.vbripress.com/amp

\begin{abstract}
PPY, PANI and PPY-PANI-ZnO ternary nanocomposites were synthesized in the laboratory via chemical oxidative polymerization method. The formation of PPY, PANI and corresponding change in the nanostructure with the incorporation of $\mathrm{ZnO}$ was examined by $\mathrm{X}$-ray diffraction technique (XRD). Nano-flakes like structure were formed in the PPY-PANI polymeric matrix with the embodiment of $\mathrm{ZnO}$ nanoparticles, as examined by surface topology images. Optical transmittance spectra showed the optimized reduced band gap $\sim 2.67 \mathrm{eV}$. Increased rate of electron-hole nonradiative recombination associated to blue and green bands was confirmed from PL spectra. An enhanced current density $\sim 197.75 \%$ was examined due to the significantly improved conductivity of PPY-PANI-ZnO ternary nanocomposites. This improved conductivity, reduced band gap and high electron-hole recombination rate confirmed that it can be used as electron transport layer in the OLED devices. Copyright $\odot$ VBRI Press.
\end{abstract}

Keywords: PPY-PANI-ZnO nanocomposites, chemical oxidative polymerization, PL, I-V, electron transport layer.

\section{Introduction}

Exceptionally unique characteristic of organic materials leads to the application in electronic and opto-electronic devices [1]. Polymer based organic light emitting diode is one of the most prominent application as thin film light source. It has attracted much more attention due to its many benefits such as large area luminescent display, low cost manufacturing, flexible transparent display etc. $[2,3]$. In order to enhance the lifetime, efficiency and brightness, OLED's are customized as multilayer structure appending electron transport layer, emission layer and hole transport layer $[\mathbf{4}, \mathbf{5}]$. The reflectance and transmittance of electron and hole are due to electrodes (cathode and anode). Inserted electrons and holes recombine in the emissive layer and produce excitons [6].

The embodiment of the inorganic particles into polymeric pattern can provide high performance novel materials which have enhanced structural, optical and electrical properties. Many researchers have evolved various conductive polymers with polyaromatic backbone including polyindole, polypyrrole, polythiophene, polyaniline etc. [7]. Among these various polymers polypyrrole and polyaniline have received huge interest due to their optical and electrical properties used in various opto-electronic applications. Both the polymers have high conductivity, environment stable, easy to synthesize and high electron mobility [8]. Whereas, $\mathrm{ZnO}$ nanoparticle have received much more attention in last two-three decades. $\mathrm{ZnO}$ is direct wide band gap semiconductor $(3.37 \mathrm{eV})$ and has exceptional excitation binding energy $\sim 60 \mathrm{MeV}$. This wide band gap is favorable for the use of $\mathrm{ZnO}$ to gather high-energy photons (UV light). $\mathrm{ZnO}$ has 90\% transparency in visible region. It has also high electron transfer capability. These attractive physical and optical properties make it potential oxide for optoelectronic devices $[\mathbf{9 , 1 0}]$. In above context, $\mathrm{ZnO}$ is the successful candidate for the incorporation in PPY-PANI polymeric pattern. PPY-PANI based $\mathrm{ZnO}$ has high electron mobility as well as high electron hole recombination rate.

Many researchers have developed the investigation on the polymeric based $\mathrm{ZnO}$ nanocomposites. Samzadeh-Kermani et al. reported the synthesis and characterization of PVA-PANI-ZnO ternary nanocomposites prepared via In-situ polymerization method for bactericidal properties [11]. Khan et al. studied the synthesis and characterization of the PANI$\mathrm{ZnO}$ nanocomposites. They also reported the stability in term of DC electrical conductivity for ammonia vapor detection application [12]. Chougule et al. reported the effect of CSA (camphor sulfonic acid) doping on PPY$\mathrm{ZnO}$ hybrid nanocomposite for the opto-electronic application [13]. In this present work, we report the concentration dependent optical, structural and electrical performance PPY-PANI-ZnO ternary nanocomposites for the application in OLED devices as electron transport materials synthesized via chemical oxidative polymerization method. 


\section{Experimental}

\section{Materials}

Pyrrole monomer (molecular weight $=67.09 \mathrm{~g} / \mathrm{mol}$ ) (Merck) with $99.99 \%$ pristine, aniline monomer (molecular weight $=$ 93.13) $($ Merck), Ammonium persulfate $($ molecular weight $=228.18 \mathrm{~g} / \mathrm{mol})($ Merck$)$ and as synthesized $\mathrm{ZnO}$ nanoparticle were procured for the synthesis process. Deionised water was used as solvent during chemical oxidative polymerization process.

\section{Synthesis of polyaniline (PANI)}

$2 \mathrm{ml}$ aniline monomer was added dropwise in $1.5 \mathrm{~mol}$ of HCL in $75 \mathrm{ml}$ distilled water and the solution was stirred constantly. Afterwards, $4 \mathrm{~g}$ ammonium persulfate as an oxidant was dissolved in $25 \mathrm{ml}$ double distilled water and then added dropwise into the above solution. For the polymerization, the solution was maintained at temperature range of $0-5^{\circ} \mathrm{C}$ with vigorous starring for 4 hours. The obtained dark green precipitate was collected and washed with 3 times distilled water and HCL respectively to remove the excess oxidant and monomer. Lastly collected precipitate was dried in vacuum oven at $70{ }^{\circ} \mathrm{C}$ for $24 \mathrm{~h}$.

\section{Synthesis of PPY-PANI-ZnO ternary nanocomposites}

PPY-PANI-ZnO ternary nanocomposites were successfully prepared via chemical oxidative polymerization process. Firstly, $0.1 \mathrm{M}$ of pyrrole monomer was dissolved in $75 \mathrm{ml}$ of distilled water. Afterwards $\mathrm{ZnO}$ was added in varying concentration $(5 \%, 10 \%$ and $15 \%)$ and exact half amount of PANI in comparison to pyrrole monomer was also added in above solution. Moreover, $0.1 \mathrm{M}$ of ammonium persulfate (APS) was dissolve in the $25 \mathrm{ml}$ of distilled water and added dropwise in the above monomer solution. For the polymerization whole solution setup was maintained at temperature range of $0-5^{\circ} \mathrm{C}$ under vigorous starring for $4-5 \mathrm{~h}$. The obtained dark blackgreen precipitate was washed with distilled water and ethanol respectively. Finally, the collected precipitate was dried in vacuum chamber at $70{ }^{\circ} \mathrm{C}$ for 24 hours. The schematic representation for the synthesis of PPY-PANI-ZnO ternary nanocomposites has been shown in Fig. 1.

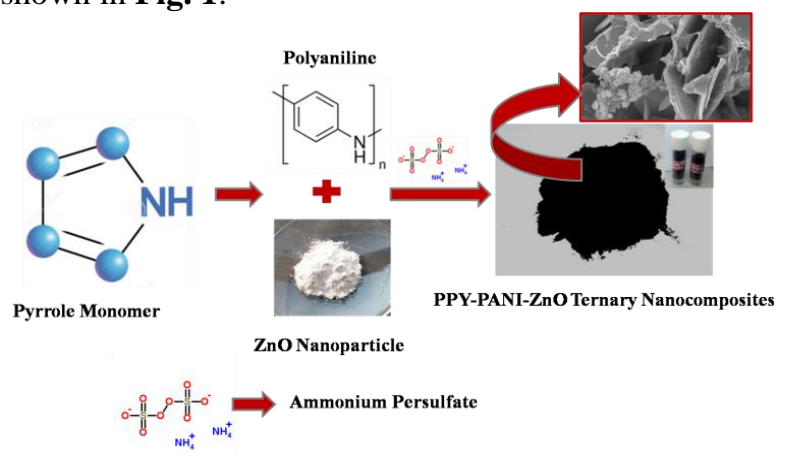

Fig.1. Schematic diagram for the synthesis of PPY-PANI-ZnO ternary nanocomposites.

\section{Characterization}

The as synthesized PPY, PANI and PPY-PANI-ZnO ternary nanocomposites were characterized by X-ray diffractometer (XRD-GBCMMA, $\mathrm{Cu}-\mathrm{K} \alpha$ radiation, $\lambda=1.5406 \AA$ ) in the $2 \theta$ angular range $10^{\circ}-65^{\circ}$. The surface topology and EDX analysis of the ternary nanocomposites were studied by Field emission scanning electron microscopy (ZEISS, SUPRA-55) and Energy Dispersive Microanalysis (Oxford Liquid Nitrogen free SDD X MAX 50 EDS) respectively. Optical transmittance spectrum obtained by UV-Vis spectroscopic technique (CARY-5000) in the wavelength range of $200 \mathrm{~nm}-800 \mathrm{~nm}$. Photoluminescence (PL) spectroscopy was performed by fluorescence spectrophotometer (Hitachi F-2500 Fluorescence Spectrophotometer) at the excitation wavelength of $320 \mathrm{~nm}$ in the emission range of $300 \mathrm{~nm}$ $600 \mathrm{~nm}$. The current-voltage measurements of these ternary nanocomposites were recorded using source meter (KEITHLEY 2400).

\section{Results and discussion}

\section{XRD analysis}

XRD spectra of Pure PPY, PANI and PPY-PANI-ZnO nanocomposites have been shown in the Fig. 2a Fig. 2e. Broad diffraction peak located at 23.15 for PPY in Fig. 2a indicating amorphous nature. In Fig. 2b, diffraction spectrum of PANI showed the peaks at $2 \theta=23.66^{\circ}$ assigned to the periodicity perpendicular to the polymer chains, revealing the degree of amorphous nature [14].

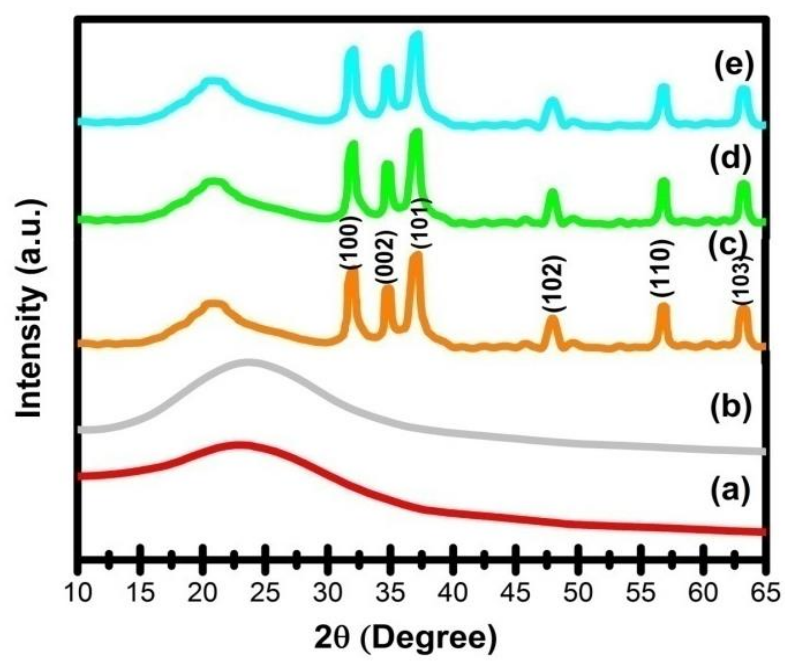

Fig. 2. XRD pattern for (a) PPY, (b) PANI, (c-e) PPY-PANI-ZnO nanocomposites of varying concentration.

Fig. 2c - Fig. 2e indicates the diffraction pattern of PPY-PANI-ZnO nanocomposites comprised a broad peak for polymers at $21^{\circ}$ and sharp peaks at an angle $2 \theta=31.99^{\circ}, 34.94^{\circ}, 36.76^{\circ}, 47.86^{\circ}, 57.10^{\circ}$ and $63.16^{\circ}$ corresponding to $\left(\begin{array}{lll}1 & 0 & 0\end{array}\right),\left(\begin{array}{lll}0 & 0 & 2\end{array}\right),\left(\begin{array}{lll}1 & 0 & 1\end{array}\right),\left(\begin{array}{lll}1 & 0 & 2\end{array}\right),\left(\begin{array}{lll}1 & 1 & 0\end{array}\right)$ and ( $\left.\begin{array}{lll}1 & 0 & 3\end{array}\right)$ for $\mathrm{ZnO}$ nanoparticle, well matched with 
JCPDS database (Card no. 36-1451) confirmed the semi-crystalline nature. The average crystallite size of nanocomposites was calculated by Debye Scherrer's formula $\left(\boldsymbol{D}=\frac{\boldsymbol{K} \lambda}{\boldsymbol{B} \boldsymbol{\operatorname { C o s } \boldsymbol { \theta }}}\right)$ to be $45 \mathrm{~nm}$ and it decreased with increase in $\mathrm{ZnO}$ content. The average inter chain separation $\left(\boldsymbol{S}=\frac{\mathbf{5} \boldsymbol{\lambda}}{\mathbf{8} \boldsymbol{\operatorname { S i n } \boldsymbol { \theta }}}\right)$ was calculated $\sim 7.25 \AA$. The diffraction peaks of corresponding nanocomposites confirmed the formation of PPY-PANI-ZnO ternary nanocomposites.

\section{FESEM analysis}

In order to investigate the surface topology and morphology, FESEM analysis has been carried out. Fig. 3 showed the FESEM images of pristine PPY, PANI and PPY-PANI-ZnO ternary nanocomposites of varying concentration. Pristine PPY showed the spherical ball type structure while pristine PANI exhibited agglomerated nanofibers. FESEM images of ternary nanocomposite showed, with the addition of $\mathrm{ZnO}$ content in ternary nanocomposite, nano-flakes structure formed and it also increased the aggregation of the nanoparticle due to the absorption of $\mathrm{H}$ atom from $\mathrm{NH}_{2}$ group of polypyrrole on the surface of $\mathrm{ZnO}$ nanoparticle. This confirmed the increment of crystallinity with the addition of the $\mathrm{ZnO}$ content. The EDX (energy dispersive X-ray spectroscopy) spectra of PPY-PANI-ZnO ternary nanocomposite confirmed the successful embodiment of $\mathrm{ZnO}$ nanoparticle into polymeric pattern.

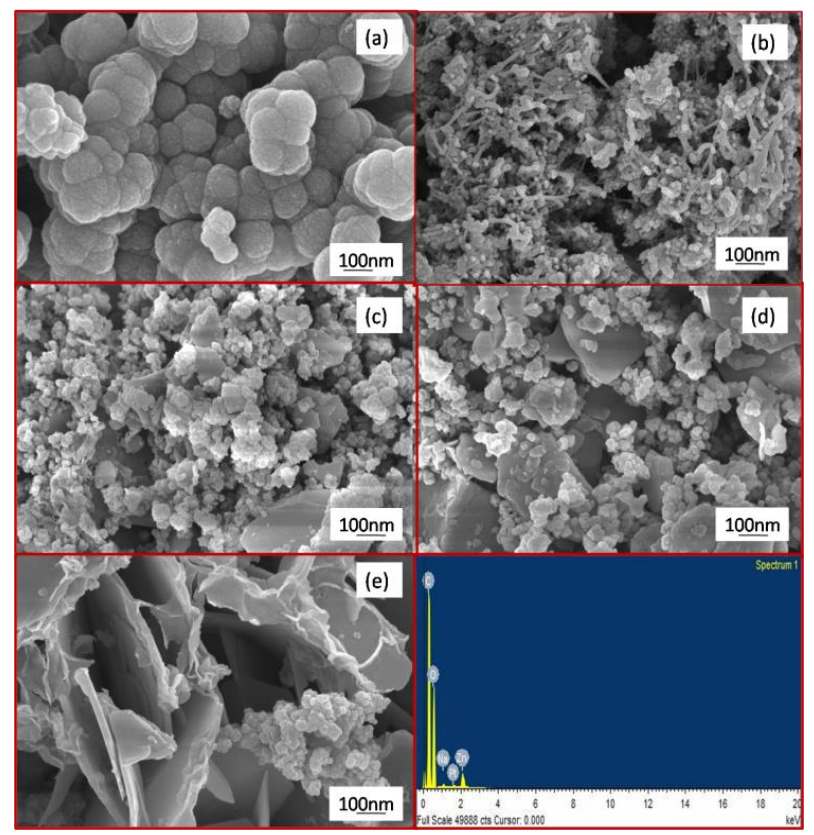

Fig. 3. FESEM images for (a) PPY, (b) PANI, (c) 5\% PPY-PANI$\mathrm{ZnO}$, (d) $10 \%$ PPY-PANI-ZnO, and (e) $15 \%$ PPY-PANI-ZnO nanocomposites and EDX spectrum of nanocomposite sample.

\section{$U V$-Vis transmittance spectroscopy}

The transmittance spectra of pristine PPY, PANI and PPY-PANI-ZnO ternary nanocomposites were reported using double beam UV-Visible spectrometer and shown in Fig. 4. These ternary films had excellent transmittance and very low absorption and reflectance. It showed $80 \%$ transparency in the visible region. There was almost negligible significant change in the transmittance spectrum observed for pure PPY, PANI and their nanocomposites in the wavelength range $200 \mathrm{~nm}-250 \mathrm{~nm}$ due to the high ultraviolet light absorption (low transmittance). This low transmittance corresponded to the PPY and PANI organic fillers. The transmittance spectrum of ternary nanocomposites was increased from the $350 \mathrm{~nm}$, probably due to the reduced absorption of $\mathrm{ZnO}$ nanoparticle. The optimized higher band edge transmittance was observed for the $10 \%$ PPY-PANI-ZnO nanocomposites around $490 \mathrm{~nm}$ associated to blue-green region.

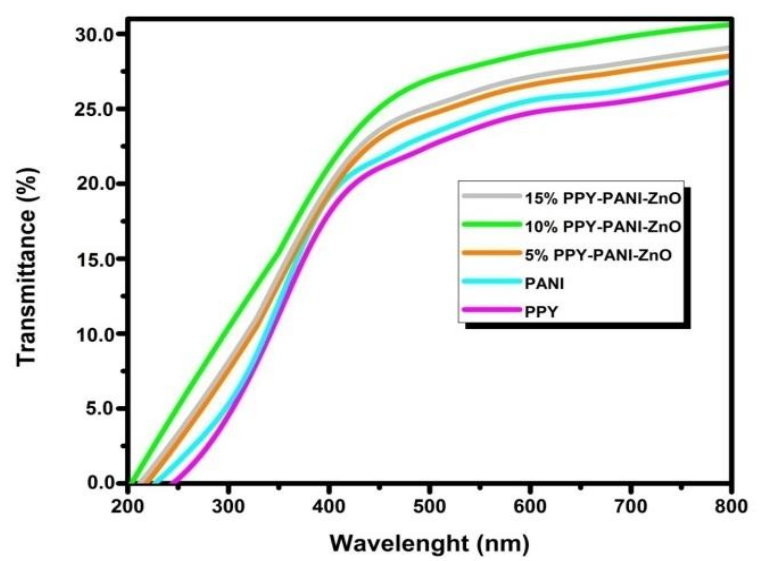

Fig. 4. UV-Visible transmittance spectra for PPY, PANI and PPYPANI-ZnO nanocomposites of varying concentration.

\section{Band gap measurement}

The optical band gap of the PPY, PANI and PPYPANI-ZnO ternary nanocomposites was determined from the transmittance spectrum shown in Fig. 5. The frequency dependent absorption coefficient stated in following relation;

$$
(\alpha h v)^{1 / n}=A\left(h v-E_{g}\right)
$$

where, $\alpha$-absorption coefficient, ho- incident photon energy, A- constant, $E_{g}$ - band gap and n- nature of electronic transition. The absorption coefficient $\alpha$ is calculated using Beer's Lambert law stated in following relation.

$$
\alpha=\frac{\ln \left(\frac{1}{T}\right)}{d}
$$

where, $\mathrm{T}$ represents transmittance of the films and $\mathrm{d}$ represents the film thickness. The as prepared PPY, PANI and PPY-PANI-ZnO ternary nanocomposites were deposited in the glass substrate by spin coating technique. The direct allowed plot between $(\alpha h v)^{2}$ and ho of ternary nanocomposites revealed the huge influence due to incorporation of $\mathrm{ZnO}$ nanoparticle. It was observed that with the increase in $\mathrm{ZnO}$ concentration in PPY host matrix, optical energy band gap decreased due to the formation of new level in the 
energy gap, which begun to move the electron from valance band through new level to conduction band. Subsequently, band gap decreased and conductivity increased. The optimized band gap of 10\% PPY-PANI$\mathrm{ZnO}$ ternary nanocomposites was $\sim 2.67 \mathrm{eV}$. The reduction in band gap led to increased p-type conductivity.

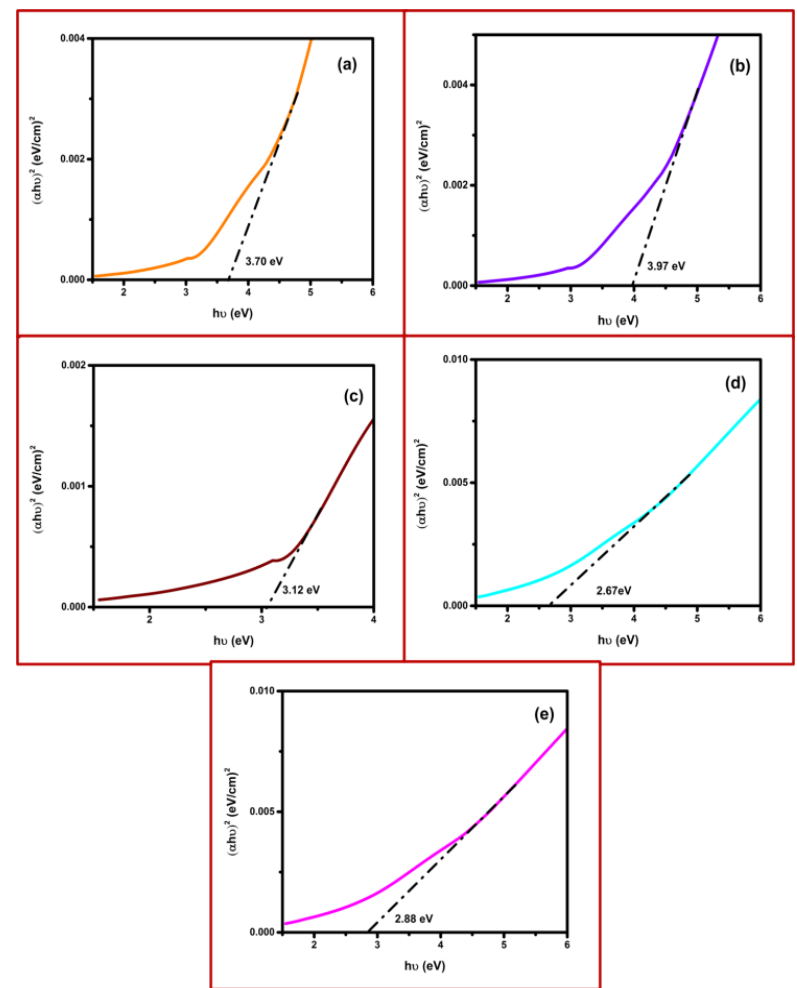

Fig. 5. Optical band gap for (a) PPY, (b) PANI, (c) 5\% PPY-PANI$\mathrm{ZnO}$, (d) $10 \%$ PPY-PANI-ZnO, and (e) $15 \%$ PPY-PANI-ZnO nanocomposites.

\section{Photoluminescence analysis}

The PL spectra emission spectra for PPY-PANI-ZnO ternary composites at $320 \mathrm{~nm}$ excitation energy have been shown in Fig. 6. There were two distinguishable emission peaks observed at $450 \mathrm{~nm}$ and $495 \mathrm{~nm}$ due to nonradiative recombination of electron-hole pair in the nanocomposite film. These exhibited the luminescence under blue and green region respectively. The additional peak recorded at $465 \mathrm{~nm}$ was attributed to deep energy states caused by impurities of $\mathrm{ZnO}$ filler in the ternary nanocomposite. The emission peak observed at $450 \mathrm{~nm}$ due to the phonon assisted transition accredit due to recombination between the electrons in deep defect level and holes in valance band of PPY-PANI$\mathrm{ZnO}$ ternary nanocomposites $[\mathbf{1 5}, \mathbf{1 6}]$. The green band occurred due non-radiative recombination of electron in the conduction band and holes trapped by the site of oxygen $\left(\mathrm{O}_{2}\right)$ vacancy of $\mathrm{ZnO}$ fillers. PL spectra showed the optimum luminance intensity for the $10 \% \mathrm{PPY}$ PANI-ZnO ternary nanocomposites due to the high electron-hole recombination rate. The increase rate of electron-hole recombination is well in favor of OLED's as electron transporting layer.

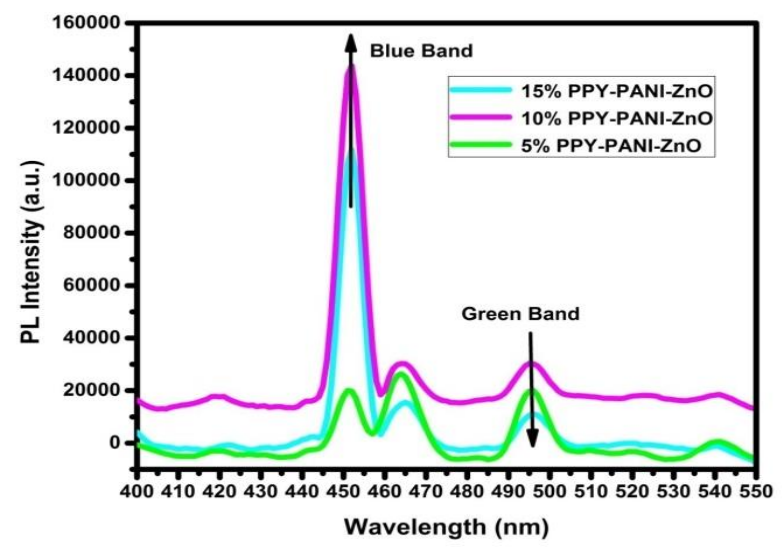

Fig. 6. PL spectra for PPY-PANI-ZnO nanocomposites of varying concentration.

\section{$J-V$ measurement}

Fig. 7 showed the J-V Characteristics of Pristine PPY, PANI and PPY-PANI-ZnO ternary nanocomposites. The measurement was taken at room temperature in the potential range of $-8.0 \mathrm{~V}$ to $+8.0 \mathrm{~V}$. All recorded data clearly indicates the linear relation between current and voltage which confirmed the ohmic nature. Current density was increased with the increase $\mathrm{ZnO}$ content and optimized current density was found for $10 \% \mathrm{PPY}$ PANI-ZnO ternary nanocomposites $\sim 7.95 \mathrm{~mA} / \mathrm{cm}^{2}$ due to the higher electron mobility in nanocomposites. An enhanced current density was recorded at $197.75 \%$ as compared to pure PANI. Based on above clarification electron-hole recombination was carried out. Embodiment of $\mathrm{ZnO}$ nanoparticle into polymeric pattern increased the minority charge carrier (hole) as compared to the majority charge carrier (electron). Consequently resistivity decreased and recombination rate was higher in $10 \%$ PPY-PANI-ZnO nanocomposites showing the p-type behavior which was in good agreement to the result of UV-Vis absorption spectroscopy. These electrical properties confirmed that this ternary nanocomposite can be used as electron transport layer in OLED devices.

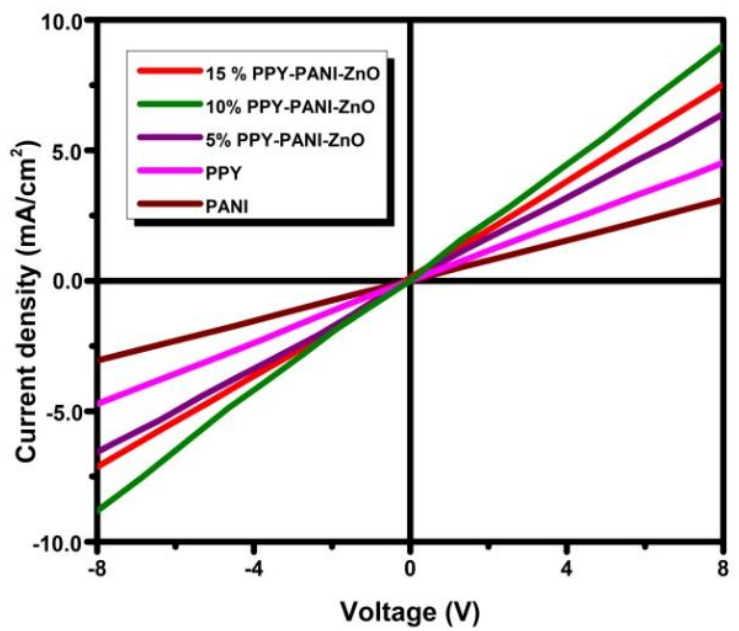

Fig. 7. J-V characteristics for PPY, PANI and PPY-PANI-ZnO nanocomposites of varying concentration. 


\section{Conclusion}

Pristine PANI, PPY and PPY-PANI-ZnO ternary nanocomposites of varying concentration were successfully synthesized via chemical oxidative polymerization method. The average crystallite size and average chain separation was $\sim 45 \mathrm{~nm}$ and $\sim 7.25 \AA$ respectively and corresponding diffracted peaks confirmed the formation of PPY-PANI-ZnO ternary nanocomposites. FESEM images showed the nanoflake structure formed due to $\mathrm{ZnO}$ nanofillers in the surface PPY-PANI. EDX analysis confirmed the successful incorporation of $\mathrm{ZnO}$ in polymeric pattern. The UV-Vis transmittance spectroscopy showed 80\% transparency in visible region and transmittance characteristic peak at $490 \mathrm{~nm}$ corresponded to bluegreen region. Optimum band gap $\sim 2.67 \mathrm{eV}$ for $10 \%$ PPY-PANI-ZnO nanocomposites. PL emission spectra showed the two identifiable peaks at $450 \mathrm{~nm}$ and $495 \mathrm{~nm}$ associated to the blue band and green band respectively. An enhance current density 197.75\% as compared to PANI neat polymer was found for the $10 \%$ PPY-PANI-ZnO ternary nanocomposites. These optical and electrical properties confirmed its application in OLED device fabrication as electron transport materials.

\section{Acknowledgements}

Authors would like to express their sincere thanks to Prof. D.C. Panigrahi, Director IIT (ISM) Dhanbad for encouragement in this communication.

\section{References}

1. Kuo, C. H.; Peng, K. C.; Kuo, L. C.; Yang, K. H.; Lee, J. H.; Leung, M. K.; Hsieh, K. H.; Chem. Mat., 2006, 18, 4121.

2. Burroughes, J. H.; Bradley, D. D. C.; Brown, A. R.; Marks, R. N.; Mackey, K.; Friend R. H.; Burn, P. L.; Holmes, A. B.; Nature, 1990, 347, 539.

3. Blom, P. W. M.; Berntsen, A. J. M.; Liedenbaum, C.; Schoo, H. F. M.; Croonen, Y.; Weijer, P. V. D.; J. Mater. Sci. Mater. Electron., 2000, 11, 105.

4. Yang, X.; Wu, F. I.; Haverinen, H.; Li, J.; Cheng, C. H.; Jabbour, G. E.; Appl. Phys. Lett. 2011, 98, 033302.

5. Meerheim, R.; Scholz, S.; Olthof, S.; Schwartz, G.; Reineke, S.; Walzer, K.; Leo, K.; J. Appl. Phys., 2008, 104, 014510.

6. Choi, H. W.; Kim, S. Y.; Kim, W. K.; Lee, J. L.; Appl. Phys. Lett., 2005, 86, 012104.

7. Gupta, K.; Jana, P. C.; Meikap, A. K.; Synth. Met., 2010, 160, 1566.

8. Lee, Y.; Kim, C.; Jang, W.; Choi, H.; Jhon, M.; Polymer, 2001, 42, 8277.

9. Ji, L. C.; Huang, L.; Liu, Y.; Thin Solid Films, 2011, 519, 3789.

10. Murugadoss, G.; J. Mate. Sci. Techn., 2012, 28, 587.

11. Samzadeh-Kermani, A.; Mirzaee, M.; Ghaffari-Moghaddam, M.; Adv. Biol. Chem., 2016, 6, 1.

12. Khan, A. A.; Khalid, M.; J. Appl. Polym. Sci., 2010, 117, 1601.

13. Chougule, M. A.; Khuspe, G. D.; Sen, S.; Patil V. B.; Appl. Nanosci., 2013, 3, 423.

14. Ali, T. M.; Padmanathan, N.; Selladura, S.; Ionics, 2015, 21, 829.

15. Reyolds, D. C.; Look, D. C.; Jogai, B.; Morkoc, H.; Solid State Commun., 1997, 101, 643.

16. Vanheusden, K.; Warren, W. L.; Seager, C. H.; Tallant. D. R.; Voigt, J. A.; Gnade B. E.; J. Appl. Phys., 1996, 79, 7983. 